\title{
Suppression of hepatic dysfunction in tenascin-X-deficient mice fed a high-fat diet
}

\author{
SHINSAKU YAMAGUCHI ${ }^{1,2}$, KOHEI KAWAKAMI ${ }^{3}$, KAZUMI SATOH ${ }^{1}$, \\ NAOKI FUKUNAGA $^{1}$, KAZUHITO AKAMA ${ }^{2}$ and KEN-ICHI MATSUMOTO ${ }^{1}$
}

${ }^{1}$ Department of Biosignaling and Radioisotope Experiment, Interdisciplinary Center for Science Research, Organization for Research and Academic Information, Shimane University, Izumo, Shimane 693-8501; ${ }^{2}$ Department of Biological Science, Shimane University, Matsue, Shimane 690-8504; ${ }^{3}$ Department of Experimental Animals, Interdisciplinary Center for Science Research, Organization for Research and Academic Information, Shimane University, Izumo, Shimane 693-8501, Japan

Received May 18, 2016; Accepted June 13, 2017

DOI: $10.3892 / \mathrm{mmr} .2017 .7052$

\begin{abstract}
Extracellular matrix glycoprotein tenascin-X (TNX) is the largest member of the tenascin family. In the present study, the contribution of TNX to liver dysfunction was investigated by administration of high-fat and high-cholesterol diet with high levels of phosphorus and calcium (HFCD) to wild-type (WT) and TNX-knockout (KO) mice. After 16 weeks of HFCD administration, the ratio of liver weight to body weight was approximately $22 \%$ higher in the HFCD-fed WT mice compared with the HFCD-fed TNX-KO mice, indicating hepatomegaly in HFCD-fed WT mice. Histological analyses with hematoxylin and eosin staining at 21 weeks revealed that hepatocyte hypertrophy in HFCD-fed TNX-KO mice was suppressed to $85 \%$ of that in HFCD-fed WT mice. By contrast, there was a 1.2-fold increase in lipid deposition in hepatocytes from HFCD-fed TNX-KO mice compared with HFCD-fed WT mice at 18 weeks, as demonstrated by Oil Red O staining. In addition, TNX-KO mice at 21 weeks and 27 weeks post-HFCD administration exhibited significant suppression of inflammatory cell infiltrate to 51 and $24 \%$ of that in WT mice, respectively. Immunofluorescence analysis for type I collagen and Elastica van Gieson staining demonstrated a clear hepatic fibrosis progression in HFCD-fed WT mice at 27 weeks, whereas hepatic fibrosis was undetected in HFCD-fed TNX-KO mice. The present findings indicated that TNX deficiency suppressed hepatic dysfunction induced by HFCD administration.
\end{abstract}

Correspondence to: Professor Ken-Ichi Matsumoto, Department of Biosignaling and Radioisotope Experiment, Interdisciplinary Center for Science Research, Organization for Research and Academic Information, Shimane University, 89-1 Enya-cho, Izumo, Shimane 693-8501, Japan

E-mail: matumoto@med.shimane-u.ac.jp

Key words: extracellular matrix, tenascin-X, liver dysfunction, high-fat and cholesterol diet, knockout mice

\section{Introduction}

The extracellular matrix (ECM), not only fills the extracellular spaces and provides structural support and tissue organization, but is also important for regulating tissue homeostasis and cell proliferation, migration, differentiation, and survival (1). In healthy tissues, the ECM provides an optimal environment for normal cell functions. However, dysregulation of ECM production and proteolysis is often associated with the onset of pathological conditions (2). Among ECM glycoproteins, the tenascin family modifies cell adhesions through their adhesive and de-adhesive properties. The tenascin family consists of 4 members in vertebrates: tenascin-C (TNC), tenascin-R, tenascin-X (TNX), and tenascin-W (3).

Non-alcoholic fatty liver disease (NAFLD) is the most common cause of chronic liver disease in Western countries (4). NAFLD includes a wide histological spectrum of liver diseases, extending from simple steatosis to the inflammatory and fibrogenic condition of nonalcoholic steatohepatitis (NASH) that may progress to end-stage liver disease (cirrhosis) and hepatocellular carcinoma (5). A previous study suggested that TNC is crucial to the development of fibrosis (6). Among the tenascin family members, the association of liver diseases with a tenascin family member has been investigated only for TNC. In the fibrotic liver, TNC is localized at connective tissue-parenchymal interface of fibrotic areas (7). Upregulation of TNC is observed in the interface between poorly differentiated hepatocellular carcinoma and invaded tissues within cancerous sinusoids (8). It is of note that El-Karef et al (9) demonstrated that TNC directly contributes to liver fibrogenesis by comparing hepatitis in WT mice and TNC-knockout (TNC-KO) mice. TNC promotes liver fibrosis by increasing procollagen synthesis through enhancement of the inflammatory responses, promotion of hepatic stellate cell (HSC) recruitment and enhancement of transforming growth factor (TGF) $-\beta$ expression (9).

TNX is the largest member of the tenascin family. Mutations in the TNX gene (TNXB) in humans have been identified in the pathogenesis of Ehlers-Danlos syndrome (EDS), a heritable connective tissue disorder characterized 
by hyperextensible skin, hypermobile joints and general connective tissue fragility $(10,11)$. Complete deficiency of TNX may lead to a recessive form of a classical type of EDS (12). Conversely, TNX haploinsufficiency is associated with the hypermobility type of EDS (13). Previous studies have revealed a function for TNX in collagen deposition (14), collagen fibrillogenesis (15), and development and maintenance of elastic fibers (16). TNX-knockout (TNX-KO) mice exhibit several features mimicking EDS, such as progressive skin hyperextensibility (14). TNX is expressed in various tissues, including skin and skeletal muscle (17). In the liver, TNX is predominantly localized to the perivascular space around central veins and is associated with blood vessels. Alcaraz et al (18) reported that TNX interacts with the latent TGF- $\beta$ complex via the C-terminal fibrinogen-like (FBG) domain of TNX, regulating the activation of the latent TGF- $\beta$ form into an active molecule. It is of note that the FGB domain of TNX induces the epithelial-to-mesenchymal transition (EMT) through latent TGF- $\beta$ activation (18).

Under pathological conditions, TNX has been demonstrated to be dramatically decreased in the tumor stroma compared with the normal dermis in a pig model of cutaneous malignant melanoma (19). Likewise, TNX was shown to be more highly expressed in low-grade astrocytomas than in high-grade astrocytomas and glioblastomas (20). However, the role of TNX in hepatic pathological conditions has not yet been elucidated. In the present study, in order to examine the role of TNX in liver dysfunction, the histological progression in WT and TNX-KO mice that were administered a high-fat and high-cholesterol diet with high levels of phosphorus and calcium (HFCD) was examined.

\section{Materials and methods}

Animals. The present study was approved by the Ethical Committee for Animal Research of Shimane University (Izumo, Japan) and all of the experimental procedures were performed according to the institutional guidelines. TNX-KO mice were generated by TNX gene targeting in murine embryonic stem cells as previously described (21). TNX-KO mice were further established by backcrossing the original TNX-KO mice into the C57BL/6 J strain for 10 generations. C57BL/6 J mice ( $n=40$; CLEA Japan, Inc., Tokyo, Japan) were used as WT mice. The mice were housed with a 12-h light-dark cycle with ad libitum access to food and water in the Department of Experimental Animals, Interdisciplinary Center for Science Research, Organization for Research and Academic Information, Shimane University. TNX-KO and WT mice [male; age, 8 weeks; ND-fed WT; $\mathrm{n}=18$ : HFCD-fed WT; n=22: ND-fed TNX-KO; n=18: HFCD-fed TNX-KO; $\mathrm{n}=22$ (weight of mice, ND-fed WT; $23.9 \mathrm{~g}(\mathrm{n}=18)$ : HFCD-fed WT; 24.6 g $(n=22)$ : ND-fed TNX-KO; 22.9 g $(n=18)$ : HFCD-fed TNX-KO; $22.8 \mathrm{~g}(\mathrm{n}=22)]$ were administered a regular chow (ND; $4.6 \%$ fat; cat. no. NMF; Oriental Yeast Co., Ltd., Tokyo, Japan) or a high-fat and high-cholesterol diet with high levels of calcium and phosphorus (HFCD; 20\% fat, $1.25 \%$ cholesterol, $1.5 \%$ calcium, $1.5 \%$ phosphorus; cat. no. F2HFD1+Ca+P; Oriental Yeast Co., Ltd.) for 9, 14, 16, 18, 21 or 27 weeks.
Alanine aminotransferase/glutamyl pyruvic transaminase (ALT/GPT) activity. Mice were anesthetized with 1-4\% isoflurane (Pfizer, Inc., New York, NY, USA) with vaporization, and blood was collected for ALT analysis in order to determine liver function. ALT activity was assayed using the Spotchem II panel-1 kit according to the manufacturer's instructions (Arkray, Inc., Kyoto, Japan).

Histological analysis. Mice were anesthetized with 1-4\% isoflurane with vaporization. The transcardial perfusion with saline in the anesthetized mice was performed, and then mice were euthanized prior to removal of the livers. The isolated livers were fixed in $10 \%$ formalin solution (Wako Pure Chemical Industries, Ltd., Osaka, Japan) for $12 \mathrm{~h}$ at $4{ }^{\circ} \mathrm{C}$, dehydrated in graded ethanol and xylene, and embedded in paraffin blocks for microtome slicing into $5 \mu \mathrm{m}$ thick sections. The sections were placed on MAS-coated slides (Matsunami Glass Ind., Ltd., Osaka, Japan), dried, and used for subsequent studies. Sections were then used for conventional hematoxylin and eosin staining and Elastica van Gieson staining, using a kit according to the manufacturer's instructions (Sigma-Aldrich; Merck KGaA, Darmstadt, Germany). Stained sections were photographed using an upright microscope (model ECLIPSE 80i; Nikon Corporation, Tokyo, Japan) with a digital camera. Quantitative analysis of hepatocyte hypertrophy was performed by imaging cytometric analysis (NIS-Elements D version 3.2; Nikon Corporation). The number of hepatocytes was counted using templates of regular squares, each of which enclosed an area of $200 \times 200 \mu \mathrm{m}$ from a total of 12 randomly-selected fields per group.

Oil Red $O$ staining. To determine lipid deposition, liver tissues obtained from the sacrificed mice from each group were fixed in $10 \%$ formalin solution for $12 \mathrm{~h}$ at $4^{\circ} \mathrm{C}$ and treated with 10 , 20 and $30 \%$ sucrose solution progressively for $4 \mathrm{~h}$ each. Then, the tissues were embedded in Tissue-Tek OTC compound (Sakura Finetek USA, Inc., Torrance, CA, USA) and frozen on dry ice. Tissue sections of $5 \mu \mathrm{m}$ thickness were cut on a cryostat (Leica Microsystems, Tokyo, Japan) and mounted on slides. The sections were stained with $0.18 \%$ Oil Red O solution (Wako Pure Chemical Industries, Ltd.) for $5 \mathrm{~min}$ at room temperature, rinsed with $60 \%$ isopropanol, and then embedded in a water-soluble mounting agent (Mount-Quick-Aqueous; Daido Sangyo Co., Ltd., Saitama, Japan). Tissue sections were photographed and quantitative analysis of stained areas from a total of 12 randomly-selected fields per group were performed using ImageJ software (version 1.48; National Institutes of Health, Bethesda, MD, USA). Oil Red O staining for each group was determined as a fold-ratio relative to the stained area measured for the control WT mice administered ND (ND-WT) at each week.

Immunofluorescence. Liver tissues were treated with 10,20 and $30 \%$ sucrose solution progressively for $4 \mathrm{~h}$ each, embedded in Tissue-Tek OTC compound, and frozen on dry ice. Sections of $5-\mu \mathrm{m}$ thickness were cut, placed on slides and fixed with $10 \%$ formalin solution for $12 \mathrm{~h}$ at $4^{\circ} \mathrm{C}$. The slides were treated with a blocking solution [5\% rabbit serum (Funakoshi Co., Ltd., Tokyo, Japan) and $0.3 \%$ Triton X-100 in PBS] and incubated with primary antibodies in $1 \%$ bovine serum albumin (Wako 
A

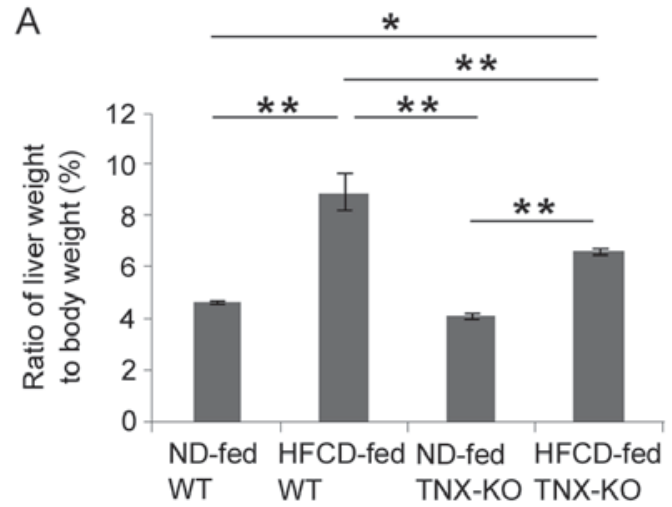

B

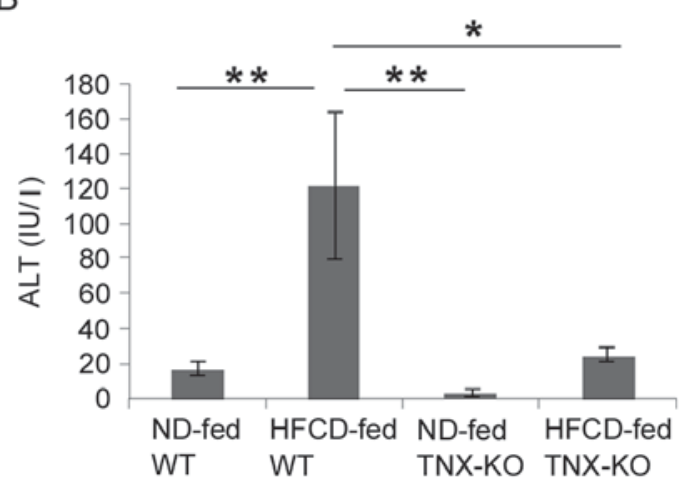

Figure 1. Effects of TNX deficiency on the liver of mice fed a HFCD. (A) WT or TNX-KO mice were fed ND or HFCD for 16 weeks, and the ratio of liver weight to body weight (\%) was measured ( $\mathrm{n}=7-8$ in each group). (B) Serum ALT levels were measured in WT and TNX-KO mice following 16 weeks of ND or HFCD diet treatment ( $\mathrm{n}=6$ in each group). ${ }^{*} \mathrm{P}<0.05$ and ${ }^{* *} \mathrm{P}<0.01$, with comparisons indicated by lines. TNX, tenascin-X; HFCD, high-fat and high-cholesterol diet with high levels of phosphorus and calcium; WT, wild-type; KO, knockout; ND, regular chow; ALT, alanine aminotransferase.

Pure Chemical Industries, Ltd., Osaka, Japan) and 0.3\% Triton $\mathrm{X}-100$ in PBS for $2.5 \mathrm{~h}$ at $4^{\circ} \mathrm{C}$, as follows: Rabbit polyclonal anti-integrin aM (also known as CD11b)/integrin aX (also termed CD11c) antibody (cat. no. bs-1014R; 1:250 dilution; Bioss, Inc., Woburn, MA, USA) and rabbit polyclonal anti-type I collagen antibody (cat. no. LB-1190; 1:250 dilution; Cosmo Bio Co., Ltd., Tokyo, Japan). For staining with the anti-type I collagen antibody, slides were heated with blocking solution at $41^{\circ} \mathrm{C}$ for 15 min during the blocking step. The sections were then washed with PBS and incubated for $1.5 \mathrm{~h}$ with Alexa Fluor 488-conjugated goat anti-rabbit secondary antibody (cat. no. 4412; 1:5,000 dilution; Cell Signaling Technology, Inc., Danvers, MA, USA). Finally, sections were washed and mounted with Prolong Gold Antifade Reagent with DAPI (Cell Signaling Technology, Inc.). Liver sections were imaged using a confocal microscope (Fluoview FV1000-D; Olympus Corporation, Tokyo, Japan), and quantified using ImageJ software (version 1.48; National Institutes of Health) from 12 randomly selected fields per group. Staining for each group was determined as a fold-ratio relative to the stained area measured for the control ND-WT mice at each week.

Statistical analysis. All data are expressed as the mean \pm standard error of the mean. Excel 2010 software (Microsoft Corporation, Redmond, WA, USA) was used for statistical analysis. Statistical significance was analyzed by analysis of variance following by Scheffé's post-hoc test. $\mathrm{P}<0.05$ was considered to indicate a statistically significant difference.

\section{Results}

TNX-KO mice exhibit suppression of HFCD-induced hepatomegaly. First, body weight gains were examined in WT and TNX-KO mice fed an ND or an HFCD. ND-fed WT, ND-fed TNX-KO and HFCD-fed TNX-KO mice exhibited similar body weight gains at 16 weeks (ND-fed WT, $31.5 \mathrm{~g}$; ND-fed TNX-KO, $32.7 \mathrm{~g}$; HFCD-fed TNX-KO, $31.8 \mathrm{~g}$ ), whereas the body weight gain of HFCD-fed WT mice (28.5 g) was significantly lower when compared with ND-fed TNX-KO mice. Food intake did not differ between WT and TNX-KO mice maintained on ND or HFCD.
Subsequently, the livers from WT and TNX-KO mice fed an ND or HFCD were examined at 16 weeks. Livers isolated from ND-fed WT and ND-fed TNX-KO mice appeared normal. However, HFCD-fed WT mice at 16 weeks had markedly swollen and ocherous livers compared with the HFCD-fed TNX-KO mice (data not shown). The ratio of liver weight to body weight was approximately $22 \%$ higher in HFCD-fed WT mice compared with HFCD-fed TNX-KO mice (Fig. 1A). Levels of serum ALT, as a marker of liver injury, were also examined in the mice at 16 weeks post-HFCD administration. ALT serum levels were significantly elevated in HFCD-fed WT mice compared with mice in all three other groups (Fig. 1B). Subsequently, histological examination was used to investigate hepatocyte hypertrophy and potential liver injury. As presented in Fig. 2A, evidence of hepatocellular injury, such as ballooning of hepatocytes was detected starting at 18 weeks post-HFCD administration in WT mice. Morphometric analysis of liver specimens demonstrated that the number of hepatocytes per constant area was significantly lower in HFCD-fed WT mice compared with HFCD-fed TNX-KO mice at 9, 18 and 21 weeks post-HFCD administration (Fig. 2B). Hepatocyte hypertrophy in HFCD-fed TNX-KO mice at 21 weeks was suppressed to $85 \%$ of that in HFCD-fed WT mice (Fig. 2B). These findings indicated that HFCD-induced hepatomegaly and liver injury were suppressed in HFCD-fed TNX-KO mice compared with HFCD-fed WT mice.

Liver steatosis in HFCD-fed WT mice. To determine the effect of HFCD administration on hepatic lipid accumulation in WT mice and TNX-KO mice, liver tissue sections from each group were stained with Oil Red $\mathrm{O}$, which is indicative of liver steatosis (Fig. 3). ND-fed TNX-KO mice exhibited a greater lipid accumulation compared with ND-fed WT mice at 9 weeks (Fig. 3). This trend continued during the 27-week period of the experimental protocol (Fig. 3). By contrast, HFCD-fed WT and TNX-KO mice tissues had increased Oil Red O staining at 9 weeks (Fig. 3). HFCD-fed WT mice displayed the greatest amount of lipid accumulation at 18 weeks; however, the quantity of lipid accumulation was gradually reduced after 18 weeks (Fig. 3). HFCD-fed TNX-KO mice; however, displayed increased lipid accumulation 
A

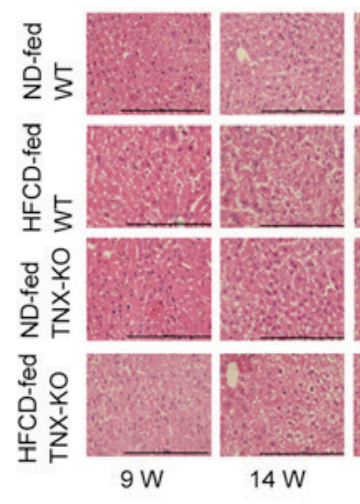

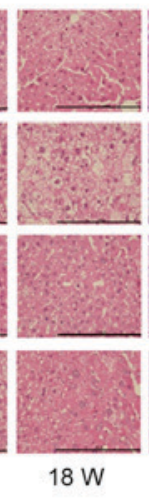

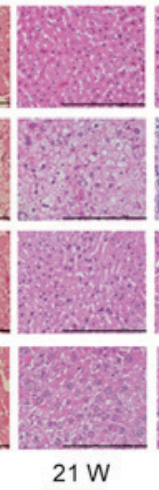

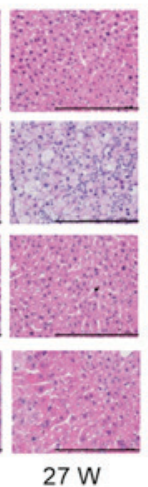

B

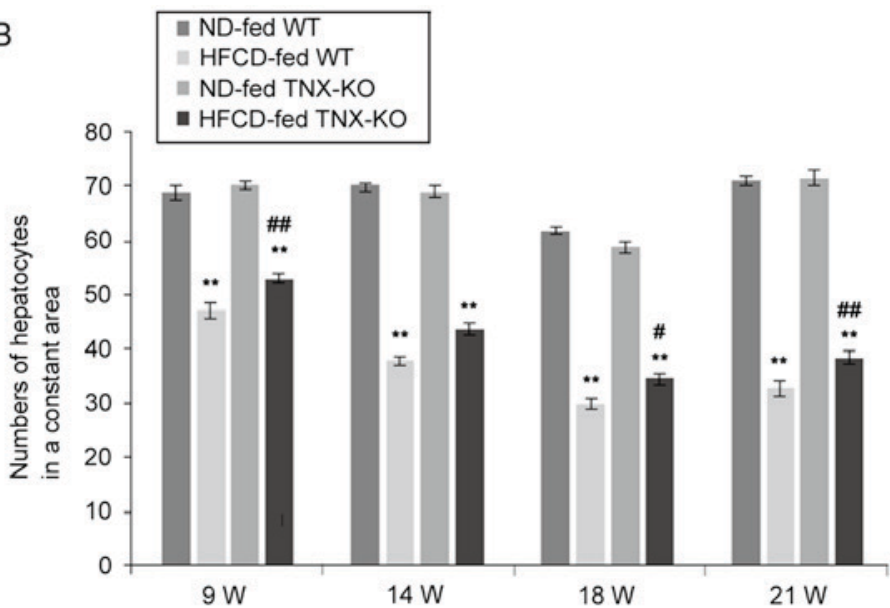

Figure 2. Effects of TNX deficiency on liver histology. (A) Representative micrographs of hematoxylin and eosin stained sections from the livers of ND-fed WT mice, HFCD-fed WT mice, ND-fed TNX-KO mice and HFCD-fed TNX-KO mice, administered the diets for 9, 14, 18 and 21 weeks. Scale bar, $200 \mu \mathrm{m}$. (B) Quantification of hepatocyte numbers per $40,000 \mu \mathrm{m}^{2}$ section area in each mouse group ( $\mathrm{n}=12$ per group). ${ }^{* *} \mathrm{P}<0.01 \mathrm{vs}$. ND-fed WT mice; ${ }^{\#} \mathrm{P}<0.05$ and ${ }^{\#} \mathrm{P}<0.01$ vs. HFCD-fed WT mice. TNX, tenascin-X; ND, regular chow; WT, wild-type; HFCD, high-fat and high-cholesterol diet with high levels of phosphorus and calcium; KO, knockout; $\mathrm{W}$, week.

A

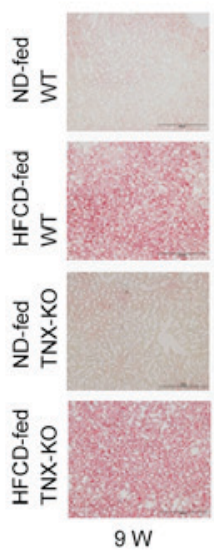

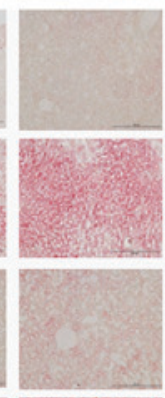
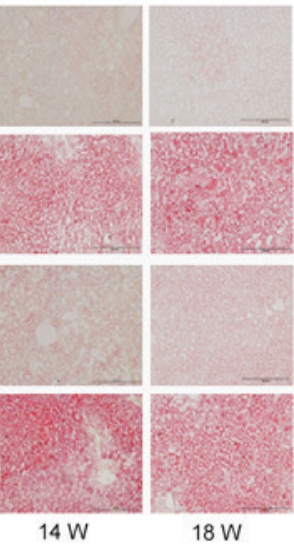

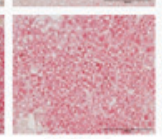

$18 \mathrm{~W}$
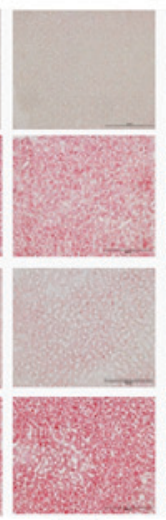

$21 \mathrm{~W}$
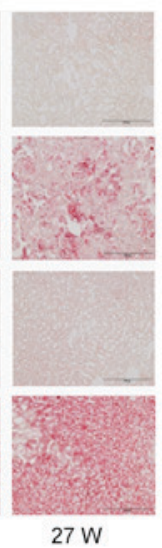

B

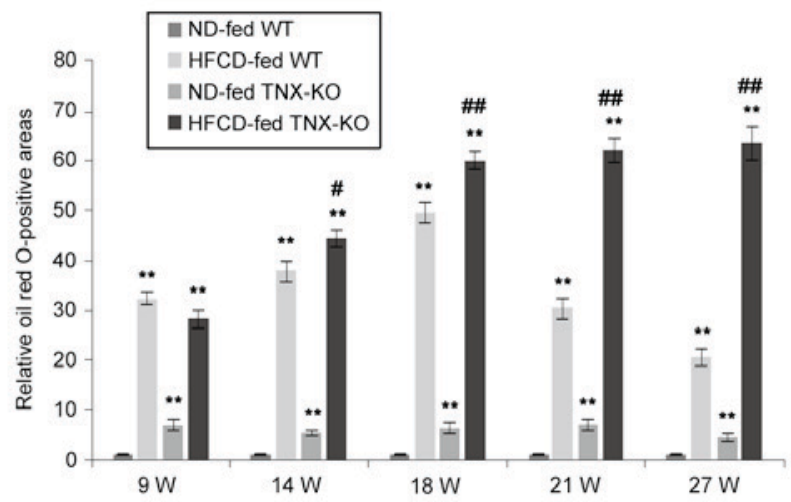

Figure 3. Effects of TNX deficiency on liver steatosis. (A) Representative micrographs of Oil Red O-stained sections from the livers of ND-fed WT mice, HFCD-fed WT mice, ND-fed TNX-KO mice and HFCD-fed TNX-KO mice, administered the diets for 9, 14, 18, 21 and 27 weeks. Scale bar, $200 \mu \mathrm{m}$. (B) Quantification of Oil Red O-positive areas as fold-change relative to the control ND-fed WT mice at each week (n=12 per group). ** P<0.01 vs. ND-fed WT mice; ${ }^{~} \mathrm{P}<0.05$ and ${ }^{\# \#} \mathrm{P}<0.01$ vs. HFCD-fed WT mice. TNX, tenascin-X; ND, regular chow; WT, wild-type; HFCD, high-fat and high-cholesterol diet with high levels of phosphorus and calcium; KO, knockout; W, week.

throughout the 27 weeks of the experimental protocol (Fig. 3). At 18 weeks, there was a 1.2-fold increase in lipid deposition in hepatocytes from HFCD-fed TNX-KO mice compared with HFCD-fed WT mice (Fig. 3).

Inflammatory cell marker infiltration is reduced in liver tissues of HFCD-fed TNX-KO mice. To further investigate a potential prevention of HFCD-induced hepatic dysfunction in TNX-KO mice, immunofluorescence analysis was performed on liver tissue sections for the inflammatory cell marker CD11b/c. Positive staining for CD11b/c was detected in liver sections from WT mice at 27 weeks post-HFCD, whereas CD11b/c staining in livers from TNX-KO mice was not evident (Fig. 4A). Quantification of CD11b/c-positive areas demonstrated that the average counts of CD11b/c-positive cells in liver sections from HFCD-fed TNX-KO mice at 21 weeks and 27 weeks were suppressed by 51 and $24 \%$ compared with HFCD-fed WT mice, respectively (Fig. 4B). By contrast, no infiltration of CD11b/c-positive cells was observed in ND-fed WT and TNX-KO mice (Fig. 4A). These findings indicated that TNX deficiency suppressed the HFCD-induced hepatic inflammatory response.

Suppression of liver fibrosis in HFCD-fed TNX-KO mice. As liver fibrosis is characterized by the excessive deposition of ECM proteins including type I collagen (22), the deposition of fibrillar type I collagen in the 4 groups was examined by immunofluorescence. Positive staining for type I collagen was detected in HFCD-fed WT mice at 27 weeks; however, it was not evident at any time point for the 3 remaining treatment groups (Fig. 5). Elastica van Gieson staining, a method of differential staining of collagen and other connective tissue, 
A
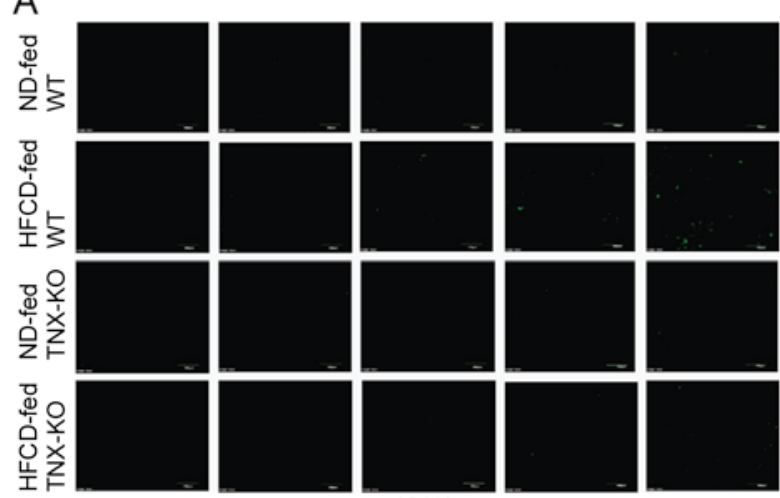

$9 \mathrm{~W}$

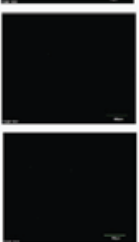

$18 \mathrm{~W}$

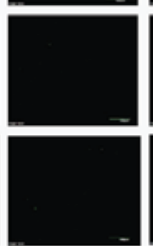

$21 \mathrm{~W}$

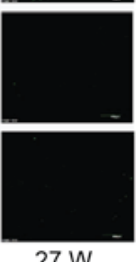

B

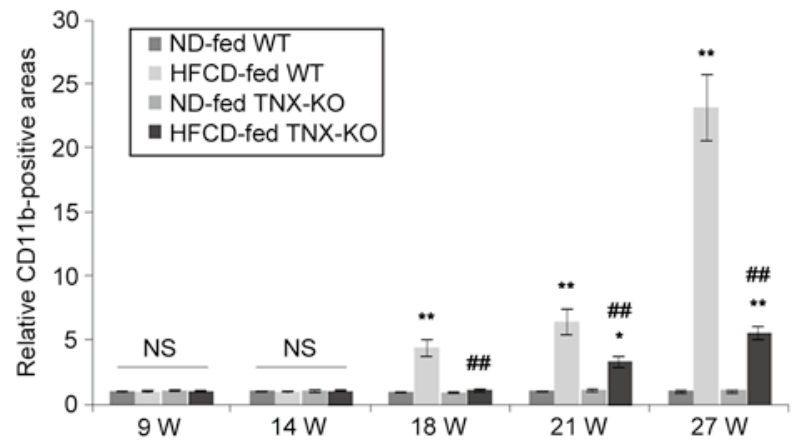

Figure 4. Effects of TNX deficiency on the liver inflammatory cell infiltrate. Liver tissue sections were prepared from ND-fed WT mice, HFCD-fed WT mice, ND-fed TNX-KO mice and HFCD-fed TNX-KO mice, administered the diets for 9, 14, 18, 21 and 27 weeks, and immune cell infiltration was evaluated by immunofluorescence for the CD11b/c immune cell marker. (A) Representative micrographs of immunofluorescence analysis for the expression of CD11b/c (green signal). Scale bar, $100 \mu \mathrm{m}$. (B) Quantification of CD11b/c-positive areas as fold-change relative to the control ND-fed WT mice at each week (n=12 per group). ${ }^{*} \mathrm{P}<0.05$ and ${ }^{* *} \mathrm{P}<0.01$ vs. ND-fed WT mice; ${ }^{\# \#} \mathrm{P}<0.01$ vs. HFCD-fed WT mice. TNX, tenascin-X; ND, regular chow; WT, wild-type; HFCD, high-fat and high-cholesterol diet with high levels of phosphorus and calcium; KO, knockout; CD11b/c, anti-integrin $\alpha \mathrm{M} /$ integrin $\alpha \mathrm{X}$; W, week; NS, not significant.

A

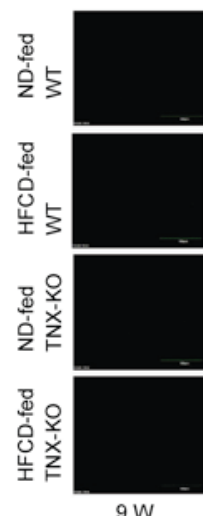

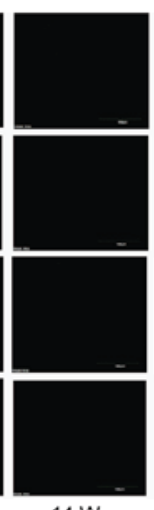

$14 \mathrm{~W}$

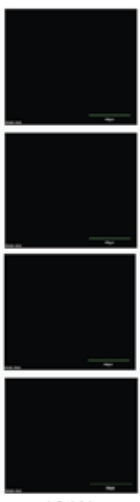

$18 \mathrm{~W}$

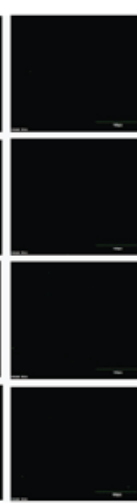

$21 \mathrm{~W}$

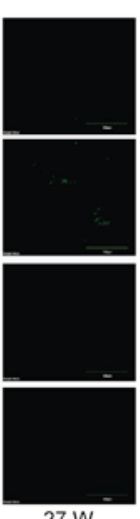

$27 \mathrm{~W}$
B

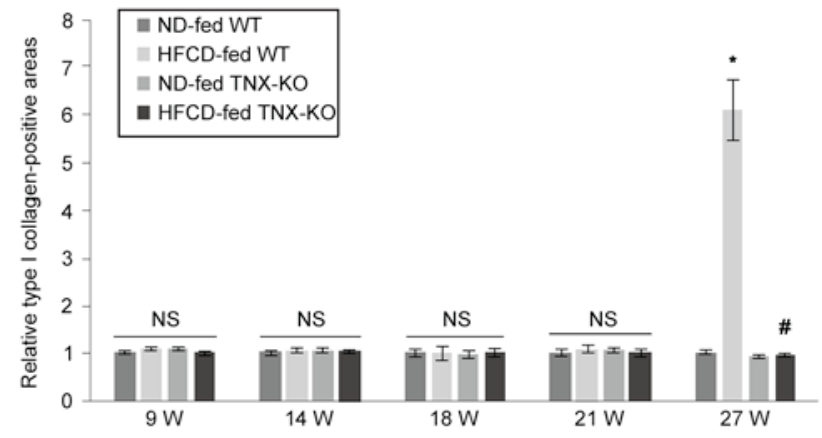

Figure 5. Effects of TNX deficiency on the liver accumulation of type I collagen. Liver tissue sections were prepared from ND-fed WT mice, HFCD-fed WT mice, ND-fed TNX-KO mice and HFCD-fed TNX-KO mice, administered the diets for 9, 14, 18, 21 and 27 weeks, and immunostained for type I collagen. (A) Representative micrographs of immunofluorescence analysis for the expression of type I collagen (green signal). Scale bar, $100 \mu \mathrm{m}$. (B) Quantification of type I collagen-positive areas as fold-change relative to the control ND-fed WT mice at each week ( $\mathrm{n}=12 \mathrm{per}$ group). ${ }^{*} \mathrm{P}<0.05 \mathrm{vs}$. ND-fed $\mathrm{WT}$ mice; ${ }^{\#} \mathrm{P}<0.05$ vs. HFCD-fed WT mice. TNX, tenascin-X; ND, regular chow; WT, wild-type; HFCD, high-fat and high-cholesterol diet with high levels of phosphorus and calcium; KO, knockout; W, week; NS, not significant.

was also performed on the liver tissue sections. As presented in Fig. 6, positive connective tissue fibers were only present in the liver tissues of HFCD-fed WT mice at 27 weeks; however, no staining was evident at any time point for the 3 remaining groups. These findings indicated that TNX deficiency suppressed the HFCD-induced liver fibrogenesis.

\section{Discussion}

To the best of our knowledge, the present study is the first to investigate the function of TNX in liver dysfunction induced by administration of HFCD. The results of histochemical analyses demonstrated that administration of HFCD induced steatosis, as well as progressive liver damage, hepatocyte ballooning, inflammatory response and hepatic fibrosis. These findings indicated that administration of HFCD to WT mice may lead to NASH. Liver dysfunction and inflammatory response in HFCD-fed TNX-KO mice was significantly less extensive compared with HFCD-fed WT mice. The results suggested that TNX may promote HFCD-induced liver dysfunction through enhancement of the inflammatory response.

As demonstrated in Fig. 3, ND-fed TNX-KO mice exhibited a greater lipid accumulation compared with ND-fed WT mice during the 27-week period of the experimental protocol. This result was consistent with a previous report, in which more lipid accumulation was detected in subcutaneous adipose tissue from TNX-KO mice compared with WT mice during ingestion of ND diets (23). Furthermore, as demonstrated in Fig. 3, maximum lipid accumulation in the liver was observed at 18 weeks post-HFCD ingestion in WT mice and subsequently gradually decreased, whereas lipid accumulation in HFCD-fed TNX-KO mice continued to increase during the 27-week period. This process may be due to decreased tissue integrity in HFCD-fed WT mice that led to leakage of lipid deposition from the damaged tissues in the liver following 18 weeks of HFCD feeding. 


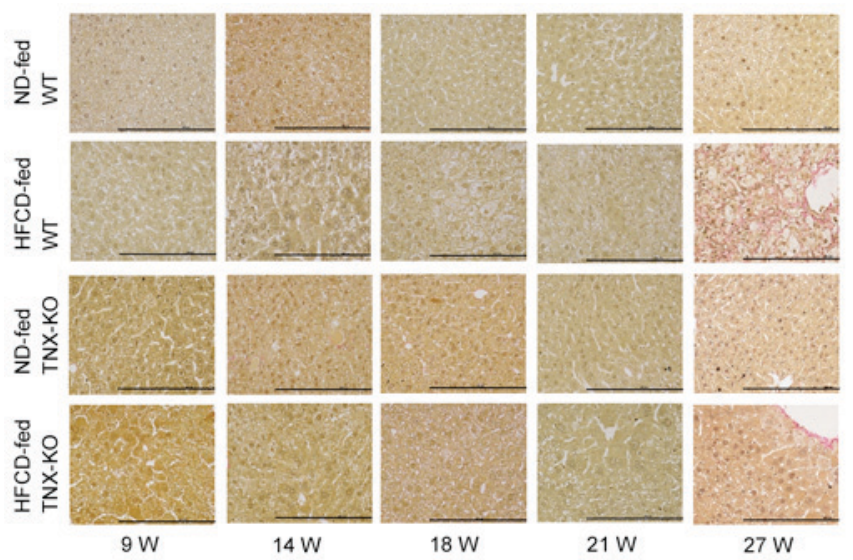

Figure 6. Effects of TNX deficiency on liver fibrosis. Representative micrographs of Elastica van Gieson-stained liver sections from ND-fed WT mice, HFCD-fed WT mice, ND-fed TNX-KO mice and HFCD-fed TNX-KO mice, administered the diets for 9, 14, 18, 21 and 27 weeks. Scale bar, $200 \mu \mathrm{m}$ TNX, tenascin-X; ND, regular chow; WT, wild-type; HFCD, high-fat and high-cholesterol diet with high levels of phosphorus and calcium; KO knockout; W, week.

It is of note that HFCD-fed WT mice did not become obese, in fact, they lost weight. However, these mice developed progressive liver damage, similar to NASH symptoms. Methionine-choline deficiency (MCD) is a well-characterized dietary rodent model of NAFLD/NASH (24). The MCD diet induces a phenotype of severe NASH, including hepatomegaly, inflammation or fibrosis, with weight loss following 8 weeks of administration $(25,26)$. By contrast, standard high-fat diet feeding generally does not lead to severe NASH despite inducing obesity or hepatic steatosis. Therefore, as the high-fat and high-cholesterol diet with high calcium and phosphorus (HFCD) used in the present study induced severe $\mathrm{NASH}$, the effect of HFCD may resemble that of MCD on the liver.

It has been previously reported that TNC, another member of the tenascin family, promotes hepatic fibrosis by augmenting the inflammatory response through hepatic stellate cell (HSC) recruitment and increased expression of TGF- $\beta$ (9). By contrast, TNC deficiency attenuates hepatic fibrosis (9). Furthermore, features similar to those of hepatic fibrosis were observed in pulmonary fibrosis, where an association between TNC and TGF- $\beta$ signaling has been previously demonstrated (27). TGF- $\beta$ is a master profibrogenic cytokine that promotes activation and differentiation of HSCs (28). Alcaraz et al (18) have reported that the FBG domain of TNX interacts with the latent TGF- $\beta$ complex, leading to its activation through a conformational change. The latent TGF- $\beta$ activation leads to an induction of the Smad signaling pathway resulting in EMT. Therefore, TNX-driven TGF- $\beta$ signaling would not be activated in TNX-KO mice. As a result, total TGF- $\beta /$ Smad signal input to cells may be weakened in TNX-KO mice, even though it is possible that other ways to activate latent TGF- $\beta$ other than TNX may exist in TNX-KO mice. This potentially attenuated TGF- $\beta$ signaling may then result in suppression of the inflammatory response and hepatic dysfunction, such as fibrosis and NASH, in the HFCD-fed TNX-KO mice. Further studies will be required in the future to investigate whether TGF- $\beta$ signaling may be involved in the progression of liver dysfunction observed in HFCD-fed TNX-KO mice.

Recently, Benbow et al (29) reported that increased expression of TNC induced by a high-fat diet enhanced inflammatory response, increased hepatocyte EMT and migration, and development of hepatocellular carcinoma through Toll-like receptor 4 (TLR4) signaling. Additionally, TNC has been previously demonstrated to be important in inflammation mediated through TLR4 in arthritic joint disease (30). In addition to the TLR4 signaling pathway, TNC enhances inflammatory response via activation of integrin $\alpha 9$ (31). Thus, since $\beta 1$-containing (32) and $\alpha 11 \beta 1$ (18) integrins have been identified as cell surface receptors of TNX, it would be interesting to determine whether TLR4-integrin signaling may contribute to suppression of hepatic dysfunction in HFCD-fed TNX-KO mice. Crosstalk between TLR4 signaling and TGF- $\beta$ signaling in hepatic fibrosis has been previously reported (33).

Complete deficiency of TNX leads to a classical type of EDS in humans (12). Patients with complete deficiency of TNX primarily exhibit hypermobile joints, hyperelastic skin and easy bruising, without atrophic scars (12). Based on the data obtained in the present study on TNX-KO mice, it would be of interest to investigate the liver functions in patients with TNX-deficient EDS. Thus far, none of patients with TNX-deficient EDS have been reported to exhibit clinical signs in liver $(34,35)$.

In conclusion, TNX deficiency suppressed hepatic dysfunction induced by HFCD administration in mice. Further studies will be required to reveal which signaling pathway is involved in the TNX-mediated hepatic dysfunction. Elucidation of the HFCD-induced TNX signaling pathway may provide potential therapeutic targets for preventing the initiation and progression of NASH. Serum TNC levels were significantly associated with necroinflammatory activity in patients with chronic hepatitis C (36). Further experimental studies will be required to evaluate the possibility that TNX may also act as a diagnostic marker and/or a therapeutic target in NASH.

\section{Acknowledgements}

The present study was supported in part by Management Expenses Grants to Shimane University.

\section{References}

1. Adams JC and Watt FM: Regulation of development and differentiation by the extracellular matrix. Development 117: 1183-1198, 1993.

2. Duarte S, Baber J, Fujii T and Coito AJ: Matrix metalloproteinases in liver injury, repair and fibrosis. Matrix Biol 44-46: 147-156, 2015.

3. Chiquet-Ehrismann R and Tucker RP: Tenascins and the importance of adhesion modulation. Cold Spring Harb Perspect Biol 3: a004960, 2011

4. Fielding CM and Angulo P: Hepatic steatosis and steatohepatitis: Are they really two distinct entities? Curr Hepatol Rep 13: 151-158, 2014.

5. Carpino G, Renzi A, Onori P and Gaudio E: Role of hepatic progenitor cells in nonalcoholic fatty liver disease development: Cellular cross-talks and molecular networks. Int J Mol Sci 14: 20112-20130, 2013.

6. Kasprzycka M, Hammarström C and Haraldsen G: Tenascins in fibrotic disorders-from bench to bedside. Cell Adh Migr 9: 83-89, 2015. 
7. Yamada S, Ichida T, Matsuda Y, Miyazaki Y, Hatano T, Hata K, Asakura H, Hirota N, Geerts A and Wisse E: Tenascin expression in human chronic liver disease and in hepatocellular carcinoma. Liver 12: 10-16, 1992.

8. Jaskiewicz K, Chasen MR and Robson SC: Differential expression of extracellular matrix proteins and integrins in hepatocellular carcinoma and chronic liver disease. Anticancer Res 13: 2229-2237, 1993.

9. El-Karef A, Yoshida T, Gabazza EC, Nishioka T, Inada H, Sakakura T and Imanaka-Yoshida K: Deficiency of tenascin-C attenuates liver fibrosis in immune-mediated chronic hepatitis in mice. J Pathol 211: 86-94, 2007.

10. Burch GH, Gong Y, Liu W, Dettman RW, Curry CJ, Smith L, Miller WL and Bristow J: Tenascin-X deficiency is associated with Ehlers-Danlos syndrome. Nat Genet 17: 104-108, 1997.

11. De Paepe A and Malfait F: The Ehlers-Danlos syndrome, a disorder with many faces. Clin Genet 82: 1-11, 2012.

12. Schalkwijk J, Zweers MC, Steijlen PM, Dean WB, Taylor G, van Vlijmen IM, van Haren B, Miller WL and Bristow J: A recessive form of the Ehlers-Danlos syndrome caused by tenascin-X deficiency. N Engl J Med 345: 1167-1175, 2001.

13. Zweers MC, Bristow J, Steijlen PM, Dean WB, Hamel BC, Otero M, Kucharekova M, Boezeman JB and Schalkwijk J: Haploinsufficiency of TNXB is associated with hypermobility type of Ehlers-Danlos syndrome. Am J Hum Genet 73: 214-217, 2003.

14. Mao JR, Taylor G, Dean WB, Wagner DR, Afzal V, Lotz JC, Rubin EM and Bristow J: Tenascin-X deficiency mimics Ehlers-Danlos syndrome in mice through alteration of collagen deposition. Nat Genet 30: 421-425, 2002.

15. Minamitani T, Ikuta T, Saito $Y$, Takebe G, Sato M, Sawa H, Nishimura T, Nakamura F, Takahashi K, Ariga H and Matsumoto K: Modulation of collagen fibrillogenesis by tenascin-X and type VI collagen. Exp Cell Res 298: 305-315, 2004.

16. Zweers MC, van Vlijmen-Willems IM, van Kuppevelt TH, Mecham RP, Steijlen PM, Bristow J and Schalkwijk J: Deficiency of tenascin-X causes abnormalities in dermal elastic fiber morphology. J Invest Dermatol 122: 885-891, 2004.

17. Matsumoto K, Saga Y, Ikemura T, Sakakura $T$ and Chiquet-Ehrismann R: The distribution of tenascin-X is distinct and often reciprocal to that of tenascin-C. J Cell Biol 125 483-493, 1994

18. Alcaraz LB, Exposito JY, Chuvin N, Pommier RM, Cluzel C, Martel S, Sentis S, Bartholin L, Lethias C and Valcourt U: Tenascin-X promotes epithelial-to-mesenchymal transition by activating latent TGF- $\beta$. J Cell Biol 205: 409-428, 2014.

19. Geffrotin C, Horak V, Créchet F, Tricaud Y, Lethias C Vincent-Naulleau S and Vielh P: Opposite regulation of tenascin- $\mathrm{C}$ and tenascin-X in MeLiM swine heritable cutaneous malignant melanoma. Biochim Biophys Acta 1524: 196-202, 2000 .

20. Hasegawa K, Yoshida T, Matsumoto K, Katsuta K, Waga S and Sakakura T: Differential expression of tenascin-C and tenascin-X in human astrocytomas. Acta Neuropathol 93: 431-437, 1997

21. Matsumoto K, Takayama N, Ohnishi J, Ohnishi E, Shirayoshi Y, Nakatsuji $\mathrm{N}$ and Ariga $\mathrm{H}$ : Tumour invasion and metastasis are promoted in mice deficient in tenascin-X. Genes Cells 6 : 1101-1111, 2001.
22. Bataller R and Brenner DA: Liver fibrosis. J Clin Invest 115 : 209-218, 2005

23. Matsumoto K, Sato T, Oka S, Orba Y, Sawa H, Kabayama K, Inokuchi J and Ariga $\mathrm{H}$ : Triglyceride accumulation and altered composition of triglyceride-associated fatty acids in the skin of tenascin-X-deficient mice. Genes Cells 9: 737-748, 2004.

24. Itagaki H, Shimizu K, Morikawa S, Ogawa K and Ezaki T: Morphological and functional characterization of non-alcoholic fatty liver disease induced by a methionine-choline-deficient diet in C57BL/6 mice. Int J Clin Exp Pathol 6: 2683-2696, 2013.

25. Machado MV, Michelotti GA, Xie G, Almeida Pereira T, Boursier J, Bohnic B, Guy CD and Diehl AM: Mouse models of diet-induced nonalcoholic steatohepatitis reproduce the heterogeneity of the human disease. PLoS One 10: e0127991, 2015.

26. Reid DT and Eksteen B: Murine models provide insight to the development of non-alcoholic fatty liver disease. Nutr Res Rev 28: 133-142, 2015.

27. Carey WA, Taylor GD, Dean WB and Bristow JD: Tenascin-C deficiency attenuates TGF- $\beta$-mediated fibrosis following murine lung injury. Am J Physiol Lung Cell Mol Physiol 299: L785-L793, 2010.

28. Hayashi H and Sakai T: Biological significance of local TGF- $\beta$ activation in liver diseases. Front Physiol 3: 12 , 2012.

29. Benbow JH, Thompson KJ, Cope HL, Brandon-Warner E, Culberson CR, Bossi KL, Li T, Russo MW, Gersin KS, McKillop IH, et al: Diet-induced obesity enhances progression of hepatocellular carcinoma through tenascin-C/toll-Like receptor 4 signaling. Am J Pathol 186: 145-158, 2016.

30. Midwood K, Sacre S, Piccinini AM, Inglis J, Trebaul A, Chan E, Drexler S, Sofat N, Kashiwagi M, Orend G, et al: Tenascin-C is an endogenous activator of Toll-like receptor 4 that is essential for maintaining inflammation in arthritic joint disease. Nat Med 15: 774-780, 2009

31. Kanayama M, Kurotaki D, Morimoto J, Asano T, Matsui Y, Nakayama Y, Saito Y, Ito K, Kimura C, Iwasaki N, et al: Alpha9 integrin and its ligands constitute critical joint microenvironments for development of autoimmune arthritis. J Immunol 182: 8015-8025, 2009.

32. Elefteriou F, Exposito JY, Garrone R and Lethias C: Cell adhesion to tenascin- $X$ mapping of cell adhesion sites and identification of integrin receptors. Eur J Biochem 263: 840-848, 1999.

33. Seki E, De Minicis S, Osterreicher CH, Kluwe J, Osawa Y, Brenner DA and Schwabe RF: TLR4 enhances TGF-beta signaling and hepatic fibrosis. Nat Med 13: 1324-1332, 2007.

34. Lindor NM and Bristow J: Tenascin-X deficiency in autosomal recessive Ehlers-Danlos syndrome. Am J Med Genet A 135: 75-80, 2005.

35. O'Connell M, Burrows NP, van Vlijmen-Willems MJ, Clark SM and Schalkwijk J: Tenascin-X deficiency and Ehlers-Danlos syndrome: A case report and review of the literature. $\mathrm{Br} \mathrm{J}$ Dermatol 163: 1340-1345 2010.

36. Tanaka H, El-Karef A, Kaito M, Kinoshita N, Fujita N, Horiike S, Watanabe S, Yoshida T and Adachi Y: Circulating level of large splice variants of tenascin-C is a marker of piecemeal necrosis activity in patients with chronic hepatitis C. Liver Int 26: 311-318, 2006. 